\title{
Microbiological Quality Assessment of Foods collected from Different Hospitals within Dhaka City
}

\author{
Fazle Alam Rabbi, ${ }^{1}$ Fazle Rabbi, ${ }^{1}$ T A Runun, ${ }^{1}$ Khadiza Zaman, ${ }^{1}$ M. Majibur Rahman, ${ }^{1}$ \\ and Rashed Noor ${ }^{1 \ddagger}$ \\ ${ }^{1}$ Department of Microbiology, Stamford University Bangladesh
}

Received 12 February 2011/Accepted 24 April 2011

\begin{abstract}
Various types of cooked food samples were collected from six different hospitals within the city of Dhaka and they were analyzed for the presence (if any) of total aerobic count (TAC), total coliform count (TCC) and total staphylococcal count (TSA) in order to determine the levels of contamination and to relate these findings to the hygiene practice of the food handlers. According to Gulf standard for microbiological criteria for foodstuff, all of the food samples exceeded the acceptable total aerobic count limit of $5 \times 10^{5}$ cfu/g while 4 out of 6 samples exceeded coliform count limit of $1 \times 10^{2} \mathrm{cfu} / \mathrm{g}$. The total coliform counts were found to be the highest in the fish $\left(1.6 \times 10^{7} \mathrm{cfu} / \mathrm{g}\right)$ and egg $\left(2.2 \times 10^{6} \mathrm{cfu} / \mathrm{g}\right)$ curry samples of hospital 1 and hospital 4 , respectively and were the lowest in the fish curry $\left(2 \times 10^{3} \mathrm{cfu} / \mathrm{g}\right)$ of hospital 4 . Staphylococcus aureus was found in all of the food samples with the highest occurrence (too numerous to count) in Dal and Rice samples from hospitals 3 and 6, respectively. On Xylose Lysine Deoxycholate (XLD) agar, no black centered colony but many colorless colonies were found which primarily indicated the absence of Salmonella spp. in those samples. In order to identify pathogenic microorganisms from food samples, a series of conventional biochemical tests were performed with 23 randomly selected isolates from MacConkey, XLD, MSA agar plates. The isolates were presumptively identified as Escherichia coli, Staphylococcus aureus, Shigella spp. and Pseudomonas spp. etc. The antibiotic susceptibility test was performed with eleven selected isolates using six commonly prescribed antibiotics (ampicillin, tetracyclines, ciprofloxacin, vancomycin, gentamicin and azithromycin). The results showed that six isolates were resistant to vancomycin, two isolates were multidrug resistant and one isolate was intermediately resistant to azithromycin. All the isolates were found to be sensitive to ciprofloxacin, and gentamicin. Based on the data, it can be suggested that adequate hygiene practices are required after cooking the foods and before serving them as they reconsider.
\end{abstract}

Hospital infections have generally been sourced from endogenous and exogenous source for long time. Endogenous infections arise from the patient himself while he is in the hospital. Hospital patients, because of their particular illness, become readily susceptible to their own commensal flora and to the potentially pathogenic microbes of their skin and mucous membranes, i.e., the secondary and/or tertiary infections.

Exogenous infections are derived from hospital environment (1). Organisms from hospital environment are generally virulent and naturally resistant to the antibiotics (2). They represent the selected microbial populations because most of the less virulent and sensitive microbes are eliminated by the antibiotic therapy in the hospital and by antibiotic fall out in the hospital environment. Exogenous infections may be transmitted by a variety of routes: (a) air borne dusts and droplets, (b) direct contact with carrier, (c) food utensils, etc. The other sources of infection greatly count on the staff and visitors to the hospital (3). So far reported, the organisms commonly responsible for hospital infections could be Staphylococcus aureus, Streptococci, Corynebacteria, E. coli, Pseudomonas, Enterotoxigenic E. coli, Shigella and Salmonella $(1,4,5)$.

\footnotetext{
${ }^{\ddagger}$ Corresponding Author. Mailing address: Dr. Rashed Noor, Dept. of Microbiology, Stamford University Bangladesh, 51, Siddeswari Road, Dhaka, Bangladesh. Phone: +88-02-8354577 (Ext-472), Fax: +88-02-8363698,
} Email: noor.rashed@yahoo.com.
Hospital food is an essential as well as unavoidable part of patient care. Good food can encourage patients to eat well, giving them the nutrients they need to recover from surgery or illness. Meals are offered to each patient three times per day, according to a fourweek cycle menu. In addition to breakfast, lunch and dinner, generally snacks are offered to each patient in the afternon by the unit staff.

Hospitals are in general thought to be the most hygienic place. However a poorly-run hospital can be responsible for any sort of food-borne illnesses and hence can serve as a reservoir of pathogens. The role of foodstuffs, contaminated by potentially pathogenic bacteria, has long been established as one of the most common causes of gastroenteritis, but the control of this condition remains a major public health problem in all communities.

Bacteria such as Salmonella and E. coli can affect any kitchen, regardless of its location, if the person preparing the food is not careful. Most bacteria found in food that leads to food poisoning are the result of improper preparation. While one would think that a hospital, among all places, would be especially mindful of this threat, the individuals responsible for preparing meals are not doctors themselves. Instead, chefs and cooks run the kitchen, like anywhere else, and can make the same mistakes that commercial cooks can make. So there is every possibility of contamination before or after cooking of a food as well as during serving. Possible sources of contamination may 
account from washing water, insects and rodents, contaminated hands or people having skin infection, hair or hair products in food, unhygienic kitchen environment, contaminated equipment, contaminated air or dust, personal hygiene, lack of adequate sanitation etc.

E. coli and S. aureus are amongst the most common pathogens found on hands $(6,7)$. It is also revealed that most hospital food handlers were carriers of $S$. aureus. Food poisoning by Staphylococcus affects hundreds of thousands of people each year (8).

In addition, the antimicrobial resistance of bacteria, isolated from food and other sources, against commonly used antibiotics has been increasing day by day (9). Considering all of these, it is most essential to maintain microbiological quality and safety of foods in the hospitals.

On the basis of these background, present study was designed to assess the microbiological quality of foods collected from different hospitals within Dhaka City by detecting the overall microbial load, identification of the pathogenic microorganisms and evaluation of the antimicrobial susceptibility tests of the identified bacteria.

\section{MATERIALS AND METHODS}

The study was confined to Dhaka City. It was a randomized cross sectional survey conducted in the city. A total of 12 food samples were collected from 6 different hospitals around Dhaka city.

About 200 gram of solid or semisolid samples were collected aseptically using sterile container and then were placed in insulated box. Food samples were then mixed with sterile saline solution and were homogenized with stomacher for 5-10 mins and serial dilutions were made up to $10^{-5}$ dilution. Bacteriological analysis were performed by standard method $(10,11)$. The microbiological condition of safety and hygiene were assayed using the methods recommended by ICMSF (10).

The quality of samples was assessed by determining aerobic plate count (APC), total coliform count (TCC), total staphylococcal count (TSC).

Inoculation and incubation. Nutrient agar plate (NA), MacConkey agar plate and Mannitol salt agar plate (MSA) were used to determine APC, TCC, TSC respectively. Spread plate technique was used to inoculate the samples on various culture media for enumeration of microbes. Six hours enrichment in selenite broth was done before inoculating the sample to the Xylose lysine deoxycholate agar (XLD) in order to determine the Salmonella and Shigella.

Bacterial count. Plate count was restricted to 30-300 colonies and plates containing more than 300 colonies were designated as too numerous to count (TNTC) and plates containing fewer than 30 colonies were designated as too few to count (TFTC). The following formula was used for enumeration:

Number of cells per $\mathrm{ml}=$ number of colonies $\mathrm{x}$ dilution factor/ volume of sample used $=\mathrm{cfu} / \mathrm{g}$

Identification of microorganisms. Identification of bacterial isolates was carried out according to Bergey's manual (12). For further confirmation several biochemical tests were performed according to the manual of methods for general Bacteriology by American Society of Microbiology(13) to identify the bacteria.

Antibiotic Susceptibility test. Antibiotic susceptibility test of the identified organisms was determined by Kirby-Bauer method using Mueller-Hinton agar medium(14). Microbes to be tested were grown in Mueller-Hinton broth medium until the turbidity was equal to 0.5 McFarland standard. Table 1 shows the antibiotics that were used in this experiment.

\section{RESULTS}

Total aerobic plate count (APC), total coliform count (TCC) and total staphylococcal count is shown in the Table 2.
TABLE 1. Potency of antibiotic discs used (Oxoid, UK)

\begin{tabular}{ll}
\hline Antibiotic used & $\begin{array}{l}\text { Potency } \\
(\mu \mathrm{g} / \text { disc })\end{array}$ \\
\hline Ampicillin (AMP) & 15 \\
Azithromycin (AZM) & 15 \\
Ciprofloxacin (CIP) & 5 \\
Gentamicin (CN) & 10 \\
Tetracycline (TE) & 30 \\
Vancomycin (VA) & 30 \\
\hline
\end{tabular}

From table 2 it is seen that all the food samples exceeded the acceptable microbiological standard and were highly contaminated. Except the rice samples, all other samples contained more than $10^{3} \mathrm{cfu} / \mathrm{g}$ coliforms which is highly unacceptable. All samples were also seriously contaminated by various species of Staphylococci which were further confirmed through biochemical tests and cultural characteristics.

TABLE 2. Aerobic plate count, total coliform count, total Staphylococcal count in different types of food collected from six different hospitals around Dhaka city.

\begin{tabular}{ccccc}
\hline Source & Food type & $\begin{array}{c}\text { APC } \\
(\mathrm{cfu} / \mathrm{g})\end{array}$ & $\begin{array}{l}\text { TCC } \\
(\mathrm{cfu} / \mathrm{g})\end{array}$ & $\begin{array}{c}\text { TSC } \\
(\mathrm{cfu} / \mathrm{g})\end{array}$ \\
\hline H-1 & Fish & $7 \times 10^{6}$ & $1.6 \times 10^{7}$ & $1.7 \times 10^{7}$ \\
& Rice & TNTC & Nil & $4 \times 10^{6}$ \\
H-2 & Dal & $1.2 \times 10^{7}$ & $7.2 \times 10^{4}$ & TNTC \\
H-3 & Vegetable & TNTC & $4.3 \times 10^{5}$ & $1.9 \times 10^{6}$ \\
H-4 & Egg & TNTC & $2.2 \times 10^{6}$ & $2.2 \times 10^{5}$ \\
& Fish & $9.8 \times 10^{6}$ & $2 \times 10^{3}$ & $1.7 \times 10^{4}$ \\
H-5 & Fish & TNTC & $1.3 \times 10^{5}$ & $6.2 \times 10^{5}$ \\
H-6 & Rice & TNTC & Nil & TNTC \\
\hline
\end{tabular}

During the study we have isolated Staphylococcus aureus, and $S$. epidermidis from the food samples. Suspected colonies of Shigella sp (isolate no 4 and 26) were isolated from fish and rice sample of hospital 1 and hospital 4, respectively. Suspected colonies of Pseudomonas spp (Isolate no 13) were isolated from vegetables collected from hospital 3. Table 3 shows the cultural characteristics and the biochemical tests of randomly selected 26 isolates. 
TABLE 3. Cultural characteristics and the biochemical tests of randomly selected twenty six (26) isolates collected from different hospital

\begin{tabular}{|c|c|c|c|c|c|c|c|c|c|c|c|c|c|c|c|c|c|c|c|}
\hline \multirow[t]{3}{*}{ Source } & \multirow[t]{3}{*}{ Sample } & \multirow[t]{3}{*}{ Medium } & \multirow{3}{*}{$\begin{array}{c}\text { Isolate } \\
\text { No }\end{array}$} & \multicolumn{5}{|c|}{ Cultural characteristics } & \multicolumn{11}{|c|}{ Biochemical test } \\
\hline & & & & \multirow[b]{2}{*}{$\frac{\mathbb{N}}{\sqrt{2}}$} & \multirow[b]{2}{*}{$\begin{array}{l}E \\
E \\
0 \\
I\end{array}$} & \multirow[b]{2}{*}{$\frac{5}{0}$} & \multirow[b]{2}{*}{ 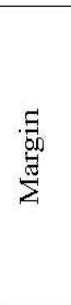 } & \multirow[b]{2}{*}{ 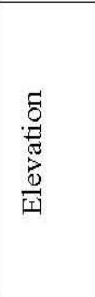 } & \multicolumn{4}{|c|}{ TSI } & \multirow[b]{2}{*}{ 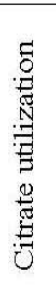 } & \multirow{2}{*}{ 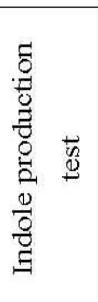 } & \multirow[b]{2}{*}{ 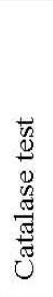 } & \multirow[b]{2}{*}{$\begin{array}{l}\vec{v} \\
\overrightarrow{0} \\
0 \\
0 \\
0 \\
\frac{\pi}{0} \\
0 \\
0\end{array}$} & \multirow[b]{2}{*}{ 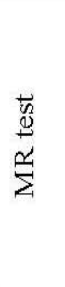 } & \multirow[b]{2}{*}{$\begin{array}{l}\frac{1}{0} \\
\stackrel{2}{5} \\
\stackrel{5}{5}\end{array}$} & \multirow[b]{2}{*}{ 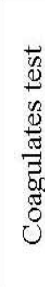 } \\
\hline & & & & & & & & & 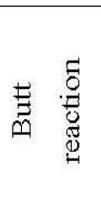 & 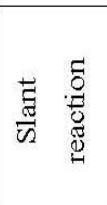 & 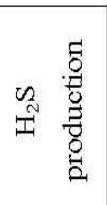 & 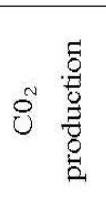 & & & & & & & \\
\hline \multirow[t]{6}{*}{$\mathrm{H}-\mathrm{l}$} & Fish & MSA & 01 & $\mathrm{~L}$ & $\mathrm{C}$ & $\mathrm{Y}$ & $\bar{E}$ & Con & ND & ND & ND & ND & - & ND & + & - & ND & ND & - \\
\hline & & & 02 & M & C & W & E & Slr & ND & ND & ND & ND & - & ND & + & - & ND & ND & - \\
\hline & & Mac & 03 & M & $\mathrm{C}$ & $\mathrm{Cl}$ & E & Slr & $\mathrm{AG}$ & $A G$ & - & - & - & - & + & - & + & - & - \\
\hline & & XLD & 04 & M & $\mathrm{C}$ & $\mathrm{Cl}$ & $\mathrm{E}$ & Slr & $\mathrm{K}$ & $\mathrm{K}$ & - & - & - & + & + & - & + & - & - \\
\hline & Rice & MSA & 05 & M & $\mathrm{C}$ & W & E & Slr & ND & ND & ND & ND & - & ND & + & - & ND & ND & - \\
\hline & & & 06 & S & C & $\mathrm{Y}$ & $\mathrm{E}$ & Con & ND & ND & ND & ND & - & ND & + & - & ND & ND & - \\
\hline \multirow[t]{4}{*}{$\mathrm{H}-2$} & Dal & MSA & 07 & M & C & $\mathrm{Y}$ & E & Con & ND & ND & ND & ND & - & ND & + & - & ND & ND & - \\
\hline & & & 08 & $\mathrm{~L}$ & C & W & $\mathrm{E}$ & Con & ND & ND & ND & ND & - & ND & + & - & ND & ND & - \\
\hline & & $\mathrm{Mac}$ & 09 & $\mathrm{~L}$ & C & $\mathrm{P}$ & $\mathrm{E}$ & Con & $\mathrm{AG}$ & $\mathrm{AG}$ & - & - & - & - & + & - & + & - & - \\
\hline & & & 10 & M & C & $\mathrm{Cl}$ & $\mathrm{E}$ & Slr & $A G$ & $\mathrm{AG}$ & - & - & - & - & + & - & - & - & - \\
\hline \multirow[t]{3}{*}{$\mathrm{H}-3$} & $\begin{array}{l}\text { Vege- } \\
\text { table }\end{array}$ & MSA & 11 & S & C & W & $\mathrm{E}$ & Con & ND & ND & ND & ND & - & ND & + & - & ND & ND & - \\
\hline & & & 12 & M & C & Y & $\mathrm{E}$ & Slr & ND & ND & ND & ND & - & ND & + & - & ND & ND & - \\
\hline & & XLD & 13 & M & C & Y & E & Slr & $\mathrm{K}$ & $\mathrm{K}$ & - & - & + & - & + & - & + & - & - \\
\hline
\end{tabular}




\begin{tabular}{|c|c|c|c|c|c|c|c|c|c|c|c|c|c|c|c|c|c|c|c|}
\hline \multirow[t]{3}{*}{ Source } & \multirow[t]{3}{*}{ Sample } & \multirow[t]{3}{*}{ Medium } & \multirow{3}{*}{$\begin{array}{c}\text { Isolate } \\
\text { No }\end{array}$} & \multicolumn{5}{|c|}{ Cultural characteristics } & \multicolumn{11}{|c|}{ Biochemical test } \\
\hline & & & & \multirow[b]{2}{*}{$\frac{\pi}{n}$} & \multirow[b]{2}{*}{ E్ } & \multirow[b]{2}{*}{$\frac{\dot{0}}{0}$} & \multirow[b]{2}{*}{ 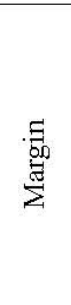 } & \multirow[b]{2}{*}{ 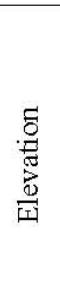 } & \multicolumn{4}{|c|}{ TSI } & \multirow{2}{*}{ 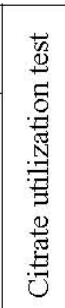 } & \multirow[b]{2}{*}{ 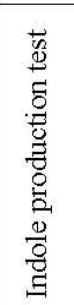 } & \multirow[b]{2}{*}{ 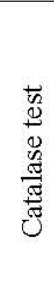 } & \multirow[b]{2}{*}{ 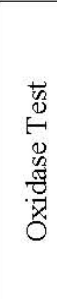 } & \multirow[b]{2}{*}{$\frac{\overrightarrow{0}}{\stackrel{\Delta}{\alpha}}$} & \multirow[b]{2}{*}{$\frac{\vec{v}}{2}$} & \multirow[b]{2}{*}{ 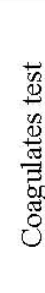 } \\
\hline & & & & & & & & & 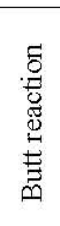 & 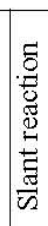 & 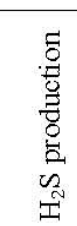 & 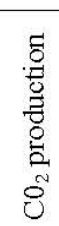 & & & & & & & \\
\hline $\mathrm{H}-4$ & Egg & MSA & 14 & M & $\mathrm{C}$ & $\mathrm{Y}$ & $\mathrm{E}$ & Con & ND & 1 & $\mathrm{NI}$ & ND & - & ND & + & - & ND & ND & - \\
\hline
\end{tabular}

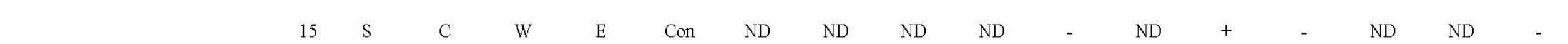

$\begin{array}{lllll}\text { MSA } & 16 & \mathrm{~L} & \mathrm{C} & \mathrm{Y}\end{array}$

E Con

ND ND ND ND

$-\quad$ ND

- ND ND

$17 \mathrm{M} \quad \mathrm{C} \quad \mathrm{W}$

Mac

$18 \quad \mathrm{~L}$

E Sl

ND ND ND ND

ND

$19 \mathrm{M} \quad \mathrm{C} \quad \mathrm{Cl}$

H-5 Fish MSA

20

C $\quad$ Y

E

AG

(n)

$\begin{array}{lllll}\text { Mac } & 22 & \text { L } & \text { C } & \text { P } \\ \text { XLD } & 23 & \text { M } & \text { C } & \text { Y }\end{array}$

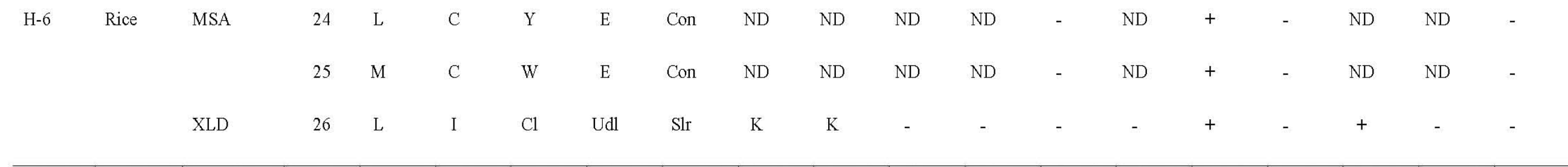

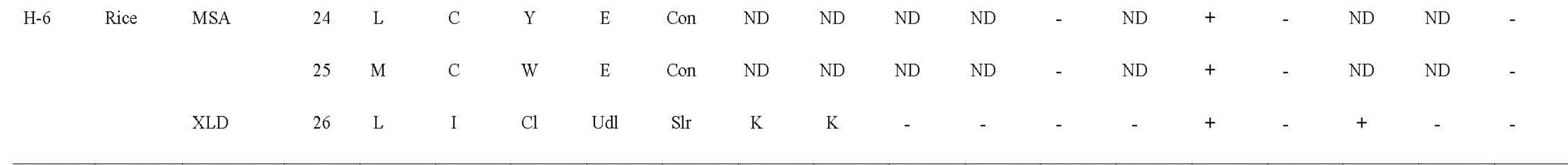

$24 \quad$ L

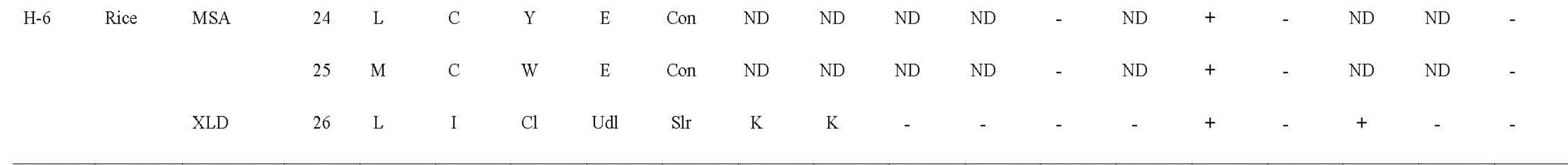

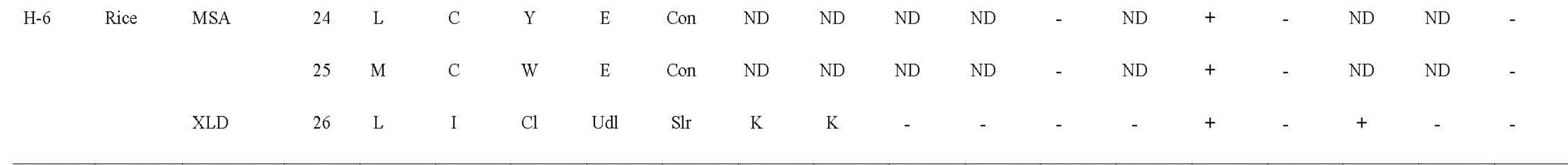

E S

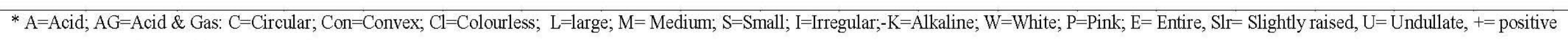

reaction, $-=$ neative reaction; $\mathrm{ND}=$ not done 
According to the above cultural and biochemical tests, the following organisms were suspected. (Table 4)

TABLE 4. Suspected organisms isolated from the hospital food.

\begin{tabular}{cccc}
\hline $\begin{array}{c}\text { Isolate } \\
\text { No }\end{array}$ & Suspected & Isolate & Suspected \\
& Organism & No & Organism \\
\hline 1 & S. aureus & 14 & S. aureus \\
2 & S. epidermidis & 15 & S. epidermidis \\
3 & E. coli & 16 & S. aureus \\
4 & Shigella sp. & 17 & S. epidermidis \\
5 & S. epidermidis & 18 & E. coli \\
6 & S. aureus & 19 & E. coli \\
7 & S. aureus & 20 & S. aureus \\
8 & S. epidermidis & 21 & S. epidermidis \\
9 & E. coli & 22 & E. coli \\
10 & E. coli & 23 & Pseudomonas sp \\
11 & S. aureus & 24 & S. aureus \\
12 & S. epidermidis & 25 & S. epidermidis \\
13 & Pseudomonas sp & 26 & Shigella sp. \\
\hline
\end{tabular}

Antibiotic susceptibility test. 11 randomly selected bacterial isolates were tested for antibiotic susceptibility by Kirby-Bauer method using 6 (ampicillin, tetracycline, ciprofloxacin, vancomycin, gentamicin and azithromycin) commonly prescribed antibiotics. The drug resistance pattern varied considerably with different drugs. The highest resistance was shown against vancomycin (6 out of 11 isolates). Moreover, 2 isolates were multidrug resistant against ampicillin, tetracyclines in addition to vancomycin and 1 isolate was intermediately resistant to azithromycin. All the isolates were found to be sensitive to ciprofloxacin, and gentamicin. Table 5 shows the antibiogram pattern of different isolates.

\section{DISCUSSION}

In the present study, we have isolated both indicator and pathogenic microorganisms and unfortunately neither of the food samples collected could meet the microbiological standard in terms of aerobic plate count (APC) or total Staphylococci count (TSC). Although there is no available epidemiological data about the risks of food-borne diseases resulting from these food supply in Bangladesh, sparse information about the risk of street-vended foods in other developing countries has been published (15).

In case of the total coliform counts, the highest occurrence was in fish $\left(1.6 \times 10^{7} \mathrm{cfu} / \mathrm{g}\right)$ and egg (2 $\left.\mathrm{x} 10^{6} \mathrm{cfu} / \mathrm{g}\right)$ curry samples of hospitals- 1 and hospital4 , respectively and the lowest was in the fish curry (2 $\mathrm{x} 10^{3} \mathrm{cfu} / \mathrm{g}$ ) of hospitals-4. Coliform count was nil in the rice samples. The presence of total coliforms led us to assume the presence of other harmful \& pathogenic microorganisms such as Salmonella spp. In our study, Salmonella spp count was nil. However considering the low sample size together with the negative data, it is not claimed that hospital food are free from Salmonella spp rather it does indicate that the prevalence of Salmonella in hospital food is very low. Besides, many colourless colonies were found on XLD agar plate that were later presumptively identified as Pseudomonas spp. and Shigella spp

On MSA agar media, huge Staphylococcal counts were found in all the samples. Besides, two types of colonies were found- yellow \& white. The organisms giving yellow colonies were assumed as $S$. aureus and the organisms giving white colonies were assumed as $S$. epidermidis. The result indicated that the food samples contaminated with Staphylococci resulted from the poor food handling practices.

Data on risk factors for foodborne diseases indicate that the majority of outbreaks result from inappropriate food handling practices (16). There are also various reports that environmental conditions

TABLE 5. Antibiogram pattern of different isolates

\begin{tabular}{|c|c|c|c|c|c|c|c|c|c|}
\hline \multirow{2}{*}{$\begin{array}{c}\text { Isolate } \\
\text { no. }\end{array}$} & \multirow{2}{*}{$\begin{array}{l}\text { Suspected } \\
\text { Organism }\end{array}$} & \multicolumn{3}{|c|}{ Antibiogram } & \multirow{2}{*}{$\begin{array}{c}\text { Isolate } \\
\text { no. }\end{array}$} & \multirow{2}{*}{$\begin{array}{l}\text { Suspected } \\
\text { Organism }\end{array}$} & \multicolumn{3}{|c|}{ Antibiogram } \\
\hline & & $\begin{array}{l}\text { Resistant } \\
\text { (R) }\end{array}$ & $\begin{array}{l}\text { Intermedi } \\
\text { ate (I) }\end{array}$ & Sensitive(S) & & & $\begin{array}{l}\text { Resistant } \\
\text { (R) }\end{array}$ & $\begin{array}{l}\text { Intermedi } \\
\text { ate (I) }\end{array}$ & Sensitive(S) \\
\hline 03 & E. coli & - & AZM & $\begin{array}{l}\text { AMP,VA,CIP, } \\
\text { CN, TE }\end{array}$ & 20 & S. aureus & - & - & $\begin{array}{l}\mathrm{AMP}, \mathrm{CIP}, \mathrm{CN}, \\
\mathrm{AZM}, \mathrm{VA}, \mathrm{TE}\end{array}$ \\
\hline 07 & S. aureus & - & - & $\begin{array}{l}\text { AMP,VA,CIP, } \\
\text { CN, TE,AZM }\end{array}$ & 21 & S. epidermidis & VA & - & $\begin{array}{c}\text { AMP,CIP,CN, } \\
\text { TE,AZM }\end{array}$ \\
\hline 12 & $\begin{array}{c}\text { S. } \\
\text { epidermidis }\end{array}$ & - & - & $\begin{array}{l}\text { AMP,VA,CIP, } \\
\text { CN, TE,AZM }\end{array}$ & 22 & E. coli & $\begin{array}{l}\text { TE,AMP, } \\
\text { VA }\end{array}$ & - & CIP,CN, AZM \\
\hline 13 & $\begin{array}{l}\text { Pseudomon } \\
\text { as sp. }\end{array}$ & VA & - & $\begin{array}{l}\text { AMP,CIP,CN, } \\
\text { TE,AZM, }\end{array}$ & 23 & Pseudomonas sp & VA & - & $\begin{array}{c}\text { AMP,CIP,CN, } \\
\text { TE,AZM }\end{array}$ \\
\hline 17 & $\begin{array}{c}\text { S. } \\
\text { epidermidis }\end{array}$ & - & - & $\begin{array}{l}\text { AMP,VA,CIP, } \\
\text { TE,CN, AZM }\end{array}$ & 26 & Shigella sp. & VA & - & $\begin{array}{l}\text { AMP,CIP,CN, } \\
\text { TE,AZM }\end{array}$ \\
\hline 19 & E. coli & $\begin{array}{l}\text { TE,AMP, } \\
\text { VA }\end{array}$ & - & CIP,CN, AZM & & & & & \\
\hline
\end{tabular}

Note: *AMP (Ampicillin), AZM (Azithromycin) CIP (Ciprofloxacin), CN (Gentamicin), TE (Tetracycline), VA (Vancomycine). 
possess significant effects on the risk of microbiological contamination (17). Practices of inadequate hygienic measures, mishandling, improper storage, inadequate cooking and above all, the unhygienic condition of the cooking premises are responsible for food-borne outbreaks (18-20). Also, the implication of food handler in food-borne disease was observed in different studies by Hall and Hauser (21). Food handlers play an important role in food safety and in the occurrence of food poisoning because they may introduce pathogens into food during production, processing, distribution and/or preparation (22). In our study, we have observed that the personal hygiene status of the hospitals was not satisfactory. The storage condition together with the kitchen environment was also very poor in every hospital. Most of the servers and cooks were also illiterate and had a very poor knowledge about personal hygiene and good manufacturing practice (GMP).

One of the major reasons of morbidity \& mortality associated with gastrointestinal infections is the antimicrobial resistance of the available drugs. In our study, we have randomly selected 11 bacterial isolates to determine the resistance against the 6 commonly prescribed antibiotics (ampicillin, tetracyclines, ciprofloxacin, vancomycin, gentamicin and azithromycin). Except ciprofloxacin and gentamicin, resistance was observed against the other four drugs. The reasons for antimicrobial resistance include inappropriate use of readily available antibiotics, reuse of antibiotics, poor implementation of infection control measures, improper disposal of hospital waste and lack of education (3, 23-25).

Finally, it can be said that most of the hospital foods are contaminated with huge load of microorganisms. Presence of coliforms \& other pathogenic bacteria indicates poor hygienic features of the foods. Patients might be taking contaminated foods with or without their knowledge. This contamination might occurred due to that most of the hospital workers are uneducated \& they are not at all concerned regarding the good hygienic practice that reduce a considerable number of pathogens from food samples. In hospitals, where a high proportion of the patients might be expected to react more severely to the ingestion of bacterial-contaminated food, it is especially important that the food prepared should be 'bacteriologically clean' and that in the place where it is handled and processed the standards of kitchen hygiene should be high. It is, therefore, essential for people who handle the foods to be properly trained on safe food handling under special care by the concerned enterprises and the governmental authorities. Lack of knowledge in food safety by the owners and servers of the restaurants promote the food contamination process unconsciously.

\section{REFERENCES}

1. McFee, R. B. 2009. Nosocomial or hospital-acquired infections: an overview. Dis. Mon. 55(7):422-38.

2. Gould, I. M. 2008.Antibiotic policies to control hospital-acquired infection. J Antimicrob Chemother. 61(4):763-5.

3. Choudhury, M. R. 2005. Hospital Infections. In Modern Medical Microbiology, (5th ed.), Vol. 43.

4. National Nosocomial Infections Surveillance (NNIS) System Report, data summary from January 1992 through June 2003, issued August 2003. Am J Infect Control, 2003. 31(8):481-98.

5. National Nosocomial Infections Surveillance (NNIS) System Report, data summary from January 1992 through June 2004, issued October 2004. Am J Infect Control 2004. 32(8):470-85.

6. Shojoei, H., J. Shooshtaripoor, and M. Amiri. 2006. Efficacy of Simple Hand-Washing in Reduction of Microbial Hand Contamination of Iranian Food Handlers. Food Research International, 2006. 39:525 529.

7. Oteri, T., and E. Ekanem. 1989. Food hygiene behavioural among hospital food handlers. Public health 103:153 -159.

8. Hazariwala, A, et al. 2002. Distribution of Staphylococcal enterotoxin genes among Staphylococcus aureus isolates from poultry and human with invasive Staphylococcal disease. Avian Diseases 46:132 -136.

9. Vikas, G., et al. 2000. Sensitivity pattern of Salmonella serotypes. BJID 6:281-282.

10. ICMSF. 1978. Sampling for Microbiological Analysis: Principles and Specific Applications. In Microorganisms in Food, 2nd ed. Chapman \& Hall, London.

11. Thatcher, F. S., and C. DS. 1968. Microorganisms in Food: Their Significance and Methods of Enumeration. University of Toronto Press, Toronto.

12. Senath, P. H. A. 1986. In Bergey's Manual of Systematic Bacteriology. W. Wilkins, Editor.

13. Gerhardt, P. 1913. General Bacteriology. American Society for Microbiology.

14. Barry, A., and C. Thornsberry. 1986. Susceptibility tests: Diffusion test procedures. In Lannette, E. H., et al. (ed.), Manual of Clinical Microbiology. ASM press, Washington, DC

15. Sami, Z. and A. Bari, 1986. Food hygiene with reference to public health. Viable bacterial counts of ready to eat foods served in Rawalpindi Islamabad. J Pak Med Assoc 36(12): 304-8.

16. Jones, T. F., and F. Angulo. 2006. Eating in Restaurants: a risk factor for food borne disease. Clinical Infection diseases 43:1324 - 1328.

17. FAO/WHO. 1974. Food-borne disease: Methods of sampling and examination in surveillance programmes. 543, W.H.O. (WHO), Editor: Geneva.

18. Bean N. H., et al. 1996. Surveillance of foodborne-disease outbreaks United States, 1988-1992. Morb. Mortal. Wkly. Rep 45(5):1-55.

19. Paulson, D. 1994. A comparative evaluation of different hand cleansers. Dairy Food Environ Sanit 14:524-528.

20. Restaino, L., and C. Wind. 1990. Antimicrobial effectiveness of hand washing for food establishments. Dairy Food Environ Sanit 10:136141.

21. Hall, H. and G. Hauser. 1966. Examination of faeces from food handlers Salmonella, Shigella, enteropathogenic Escherichia coli and Clostridium perfringens. Appl Microbiol. 14: 928-933.

22. Green, L., et al. 2005. Food service workers self reported food preparation practices: an EHS Net study. International journal of hygiene environmental health 67:27-35.

23. Hassan, M. M., et al. 2008. Pattern of medical waste management: existing scenario in Dhaka City, Bangladesh. BMC Public Health 8:36.

24. Kapil, A. 2005. The challenge of antibiotic resistance: need to contemplate. Indian J. Med. Res. 121(2):83-91.

25. Mulu, A., et al. 2006. Pattern and multiple drug resistance of bacterial pathogens isolated from wound infection at University of Gonda Teaching Hospital, Northwest Ethiopia. Ethiop. Med. J. 44(2):125-31. 